ARTICLE

https://doi.org/10.1038/s41467-019-12122-8

\title{
A novel role for the actin-binding protein drebrin in regulating opiate addiction
}

Jennifer A. Martin', Craig T. Werner', Swarup Mitra1, Ping Zhong², Zi-Jun Wang ${ }^{1}$ Pedro H. Gobira ${ }^{1,3}$, Andrew.F. Stewart ${ }^{1}$, Jay Zhang ${ }^{1}$, Kyra Erias ${ }^{1}$, Justin N. Siemian', Devin Hagarty ${ }^{4}$, Lauren E. Mueller ${ }^{1}$, Rachael L. Neve ${ }^{5}$, Jun-Xu Li ${ }^{1}$, Ramesh Chandra ${ }^{6}$, Karen C. Dietz ${ }^{1}$, Mary Kay Lobo ${ }^{6}$, Amy M. Gancarz ${ }^{4}$, Zhen Yan (1) ${ }^{2} \&$ David M. Dietz (1) ${ }^{1,7}$

Persistent transcriptional and morphological events in the nucleus accumbens (NAc) and other brain reward regions contribute to the long-lasting behavioral adaptations that characterize drug addiction. Opiate exposure reduces the density of dendritic spines on medium spiny neurons of the NAc; however, the underlying transcriptional and cellular events mediating this remain unknown. We show that heroin self-administration negatively regulates the actin-binding protein drebrin in the NAc. Using virus-mediated gene transfer, we show that drebrin overexpression in the NAc is sufficient to decrease drug seeking and increase dendritic spine density, whereas drebrin knockdown potentiates these effects. We demonstrate that drebrin is transcriptionally repressed by the histone modifier HDAC2, which is relieved by pharmacological inhibition of histone deacetylases. Importantly, we demonstrate that heroin-induced adaptations occur only in the $\mathrm{D} 1^{+}$subset of medium spiny neurons. These findings establish an essential role for drebrin, and upstream transcriptional regulator $\mathrm{HDAC2}$, in opiate-induced plasticity in the NAc.

\footnotetext{
${ }^{1}$ Department of Pharmacology and Toxicology, Program in Neuroscience, Research Institute on Addictions, The State University of New York at Buffalo, Buffalo, NY 14214, USA. ${ }^{2}$ Department of Physiology and Biophysics, The State University of New York at Buffalo, Buffalo, NY 14214, USA. ${ }^{3}$ Department of Physics and Chemistry, School of Pharmaceutical Sciences of Ribeirão Preto, University of São Paulo, Ribeirão Preto, São Paulo, Brazil. ${ }^{4}$ Department of Psychology, California State University Bakersfield, Bakersfield, CA 93311, USA. ${ }^{5}$ Gene Delivery Technology Core, Massachusetts General Hospital, Cambridge, MA 02139, USA. ${ }^{6}$ Department of Anatomy and Neurobiology, University of Maryland School of Medicine, Baltimore, MD 21201, USA. ${ }^{7}$ Department of Psychology, The State University of New York at Buffalo, Buffalo, NY 14214, USA. Correspondence and requests for materials should be addressed to D.M.D. (email: ddietz@buffalo.edu)
} 
O ne of the most difficult challenges in the treatment of opiate addiction is the life-long propensity to relapse ${ }^{1}$, contributing to the nearly 50,000 cases of overdose each year ${ }^{2}$. Drug relapse is often triggered by acute re-exposure to the drug, which also occurs in preclinical rodent models of addiction. Opiate-induced relapse is mediated by maladaptive cellular plasticity, including synaptic rewiring, in the nucleus accumbens (NAc) and several other brain regions of the mesolimbic dopamine system. The adaptions to neuronal morphology are also a hallmark of many other psychiatric disorders ${ }^{3-5}$. Synaptic strength, number of synaptic contacts, and glutamate release following abstinence from heroin have been implicated in neuronal plasticity ${ }^{6,7}$. Despite these advances, the precise molecular mechanisms responsible for the cellular and behavioral plasticity remain elusive.

The enduring adaptations observed in response to drugs of abuse, particularly in medium spiny neurons (MSNs) in the striatum, have been attributed to alterations in gene transcription regulated through epigenetic-mediated mechanisms ${ }^{8}$. Opiates ${ }^{9-11}$ and psychostimulants ${ }^{12,13}$ induce disparate molecular, transcriptional, and functional changes in the two MSN subtypes, those expressing dopamine receptor 1 (D1) or dopamine receptor 2 (D2), which project to midbrain and pallidal regions or pallidal regions only, respectively ${ }^{14-17}$.

The structural changes to neuronal dendrites, including those following exposure to drugs of abuse, are driven by actin dynamics ${ }^{18-20}$, a pathophysiological pathway common to neuropsychiatric disorders. Actin cycling is tightly controlled by robust transcription and epigenetic regulation of genes encoding cytoskeletal proteins $s^{21,22}$. One such protein is drebrin, which modulates both dendritic spine plasticity and synaptic function and is dysregulated in certain disease states ${ }^{23,24}$. However, the defining transcriptional and cell-type-specific regulators of actin dynamics that influence dendritic spine plasticity and actin homeostasis in MSNs under the influence of drugs of abuse remain largely unknown.

Gene expression is regulated, in part, through chromatin remodeling. The regulation of the chromatin state by histone acetylation drives drug-induced alterations of gene expression contributing to maladaptive behaviors ${ }^{25}$. Histone deacetylases (HDACs) are enzymes that tightly regulate this process, and HDAC inhibitors have gained momentum as potential therapeutics for neuropsychiatric diseases, such as depression ${ }^{26}$ and anxiety disorders ${ }^{27}$. However, the role of HDACs in mediating opioid-induced cellular plasticity and the therapeutic potential of HDAC inhibition in heroin abuse remain understudied.

Here, we demonstrate the epigenetic (HDAC2) and cell-typespecific (D1/D2) regulation of drebrin expression following opioid exposure, ultimately resulting in drug-induced changes in actin stability. Our results demonstrate an essential role of drebrin in heroin-induced behavioral and structural plasticity in the NAc. The studies presented here are among the first to implicate transcriptional regulation of structural plasticity, in a cell-type-specific manner, mediating susceptibility to relapse, thus providing a pathway for intervention across several domains of plasticity.

\section{Results}

Opiates alter actin dynamics in the NAc. To investigate alterations in actin dynamics in response to drugs of abuse, we trained rats to self-administer (SA) heroin (Fig. 1a), which decreases the density of dendritic spines on MSNs in the NAc shell (Supplementary Fig. 1a). We found altered actin cycling in NAc MSNs, observed as a decrease in the filamentous-to-globular actin ratio (F/G ratio) (Fig. 1b), which was driven by a decrease in F-actin pools in the NAc (Supplementary Fig. 1b, c). We next sought to determine the critical mediators of this altered actin stability.

As reduced expression of the actin-binding protein drebrin, which bundles actin filaments to maintain synaptic integrity, has been implicated in the pathogenesis of conditions marked by dendritic spine loss (i.e., Alzheimer's, schizophrenia) ${ }^{28,29}$, we assessed drebrin expression following opiate exposure. Following heroin SA, mRNA and protein levels of drebrin were decreased in the NAc (Fig. 1c and Supplementary Fig. 2), but not in the caudate putamen of the striatum (Supplementary Fig. 3a). In contrast, drebrin expression was not altered following sucrose SA (Supplementary Fig. 3b), suggesting the changes in expression may be specific to heroin exposure.

Drebrin bidirectionally regulates heroin-induced plasticity. To determine a physiological role of drebrin in the regulation of addiction-like behaviors, we utilized virus-mediated gene transfer via herpes simplex viruses (HSVs) to overexpress drebrin or green fluorescent protein (GFP) and CRISPR-Cas9 to knockdown drebrin. We first turned to a within-session dose-response paradigm, which allowed us to measure enhanced or decreased sensitivity (e.g., horizontal shifts) or can be predictive of drug vulnerability (i.e., a vertical shifts), across several doses of $\operatorname{drug}^{30}$. Specifically, vertical changes in drug reward represent an allostatic change, which has previously been demonstrates in animals based on their intrinsic vulnerability ${ }^{31}$. HSV-mediated overexpression of drebrin (Supplementary Fig. 4a, b) resulted in a vertical downward shift in heroin SA (Fig. 1d), whereas potentiation of the opiate-induced repression of drebrin by HSV-CRISPR-,drebrin (Supplementary Fig. 4c-e) resulted in an upward shift in SA (Fig. 1e), demonstrating a weakening and heightening of drug vulnerability, respectively ${ }^{31,32}$. Given the effects of drebrin expression in a model predictive of addiction vulnerability, we next sought to determine to role of drebrin in relapse-like behaviors. Drebrin overexpression in the NAc was sufficient to attenuate heroin-primed reinstatement (Fig. 1f), which was not due to an overall suppression of locomotor and operant behaviors (Supplementary Fig. 5a, b), whereas HSV-CRISPR-drebrin exacerbated drug-seeking behavior (Fig. 1g). These effects were specific for heroin-related relapse-like behaviors, as sucrose reinstatement was not altered by viral manipulation of drebrin expression (Supplementary Fig. 6a, b).

HDAC2-mediated regulation of drebrin. To determine the mechanism by which opiates downregulate drebrin expression in the NAc, we first examined the acetylation of the drebrin promoter, as histone acetylation is known to positively regulate the transcription of genes associated with synaptic plasticity ${ }^{33}$. We found that repeated morphine exposure resulted in decreased acetylation on the drebrin promoter (Supplementary Fig. 7a), which was concomitant with a decrease in drebrin expression (Supplementary Fig. 7b, c). Returning to the more translatable model of addiction-like behaviors, we sought to understand the precise mechanism by which opiate SA decreased the acetylation and expression of drebrin. Therefore, we investigated a stimulusinduced transcriptional repressor, $\mathrm{HDAC}^{34}$, as it has been highly implicated in mediating neuronal and synaptic plasticity. HDAC2 expression was upregulated in the NAc after heroin exposure (Fig. 2a) and also had increased binding along AP-1 and Smad binding element (SBE) promoter sites (Fig. 2b), which are critical sites for drug-induced transcriptional plasticity ${ }^{32,35}$. To determine a direct role of HDAC2-mediated drebrin regulation and behavioral responses, the HDAC inhibitor MI- $192^{36}$, which inhibits $\mathrm{HDAC} 2 / 3$ with higher selectivity for HDAC2 $\left(\mathrm{IC}_{50}\right.$ of 16 and $30 \mathrm{~nm}$, respectively), was bilaterally injected directly into the 
a

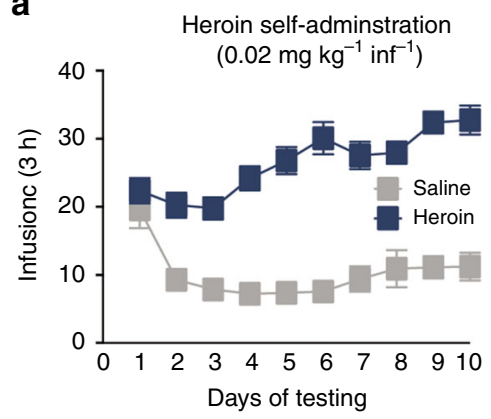

b

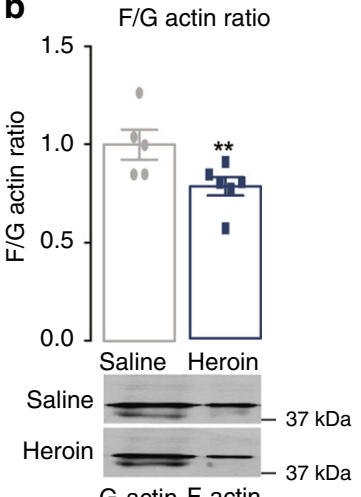

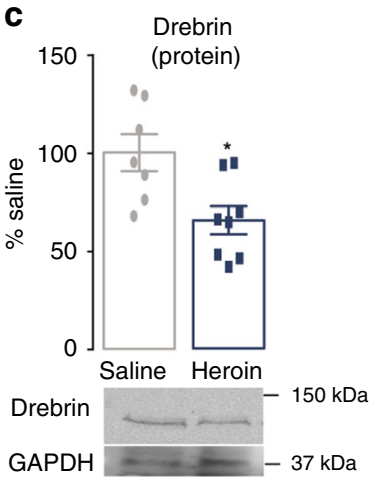

d

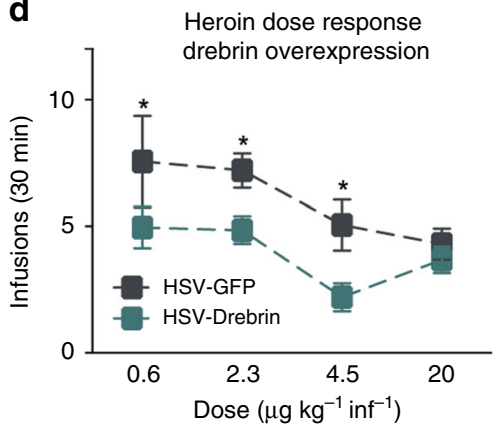

$\mathbf{f}$

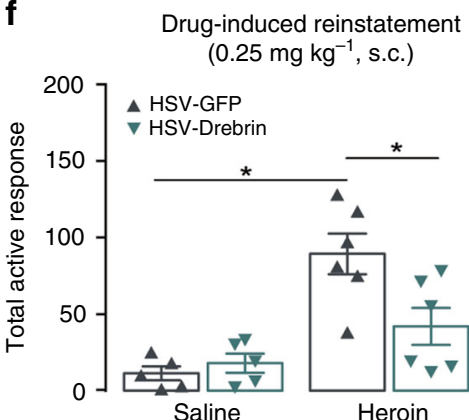

e

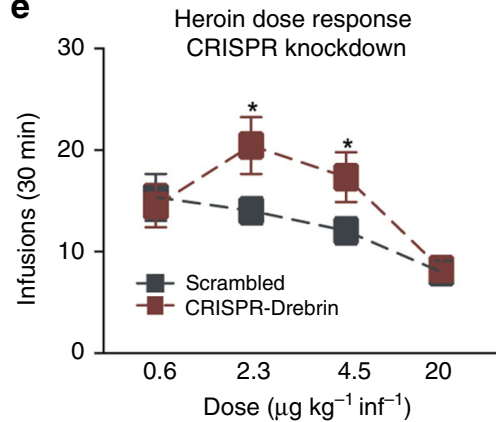

g

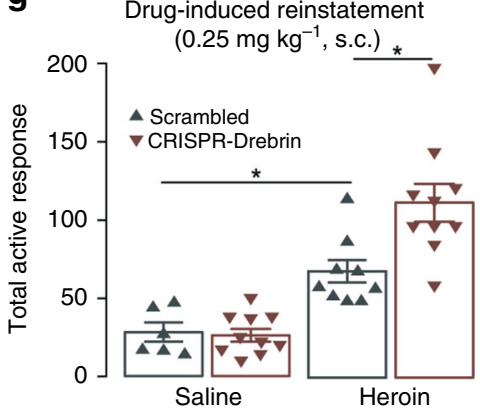

Fig. 1 Drebrin mediates behavioral plasticity in the NAc. a Mean numbers of infusions of saline (gray) or heroin (blue) self-administered over 10 days (twoway repeated-measures ANOVA: drug effect, $F_{1,41}=76.74, P<0.001$; day effect, $F_{9,369}=7.899, P<0.001$; interaction, $F_{9,369}=8.882, P<0.001, n=19-24$ per group). Actin cycling (Student's $t$-test: $t_{9}=2.632, P=0.027, n=6-7$ per group) (b) and drebrin protein levels (Student's $t$-test: $t_{13}=2.953, P=0.011$, $n=7-8$ per group) (c) in the NAc following heroin SA. Within-session heroin SA dose response ( $\mu \mathrm{g} \mathrm{kg}^{-1}$ infusion ${ }^{-1}$ [inf]) following intra-NAc injections of HSV-drebrin or HSV-GFP (two-way ANOVA: dose effect, $F_{3,72}=4.314, P<0.001$; virus effect, $F_{1,72}=10.68, P<0.01$; interaction, $F_{3,71}=0.56, P>0.05, n=$ 10-11 per group) (d) and HSV-CRISPR-Cas9 drebrin (CRISPR-drebrin) or HSV-scrambled (two-way ANOVA: dose effect, $F_{3,60}=8.796, P<0.001$; virus effect, $F_{1,60}=4.467, P<0.05$; interaction, $F_{3,60}=1.864, P>0.05, n=9-8$ per group) (e). Total active responses during drug-induced reinstatement following virus-mediated overexpression of drebrin (two-way ANOVA: drug effect, $F_{1,18}=23.68, P<0.001$; virus effect, $F_{1,18}=3.782, P=0.068$; interaction, $\mathrm{F}_{1,18}=6.63, P=0.019$; saline-GFP vs heroin-GFP, $P=0.003$; heroin-GFP vs heroin-drebrin, $P=0.017$; saline-GFP vs heroin-drebrin, $P=0.201$; $n=5-6$ per group) (f) or CRISPR-drebrin (two-way ANOVA: drug effect, $F_{1,31}=53.71, P<0.001$; virus effect, $F_{1,31}=5.937, P<0.05$; interaction, $F_{1,31}=7.17$, $P=0.01$, saline-scrambled vs heroin-scrambled, $P=0.02$; heroin-scrambled vs heroin-CRISPR, $P=0.003 ; n=6-10$ per group) (g) in the NAc. Data are expressed as means \pm SEMs. ${ }^{\star} P<0.05,{ }^{\star \star} P<0.01$

NAc. Intra-NAc inhibition of HDAC decreased HDAC2 binding at $\mathrm{AP}-1$ and SBE sites on the drebrin promoter (Fig. 2c) and increased drebrin mRNA levels (Fig. 2d). Notably, MI-192 in the NAc decreased drug-induced reinstatement (Fig. 2e). Together, these data suggest a transcriptional and epigenetic mechanism by which HDAC2 regulates drebrin expression and behavioral responses to heroin thus revealing a potential therapeutic target to reduce drug-induced relapse-like behaviors.

Drebrin in the NAc does not alter pain-related behaviors. Opiates are commonly used for their therapeutic and analgesic properties, resulting in drug tolerance with prolonged used ${ }^{37}$.
As the NAc is thought to integrate emotional and sensory information ${ }^{38,39}$, we sought to elucidate the role of drebrin in the NAc in opiate-induced pain-related behaviors and tolerance. To this end, we utilized a place escape avoidance paradigm to assess affective (emotional ${ }^{40}$ ) pain by pairing aversive stimuli with an innately preferred chamber in addition to von Frey testing to assess physical (mechanical) pain. Neither drebrin overexpression nor knockdown altered affective (Supplementary Fig. 6c, d) or physical (Supplementary Fig. 6e, f) pain. Furthermore, manipulation of drebrin expression in the NAc did not alter the induction of tolerance to the analgesic effects of opiates (Supplementary Fig. 6e, f), suggesting that drebrin in the NAc is not a 


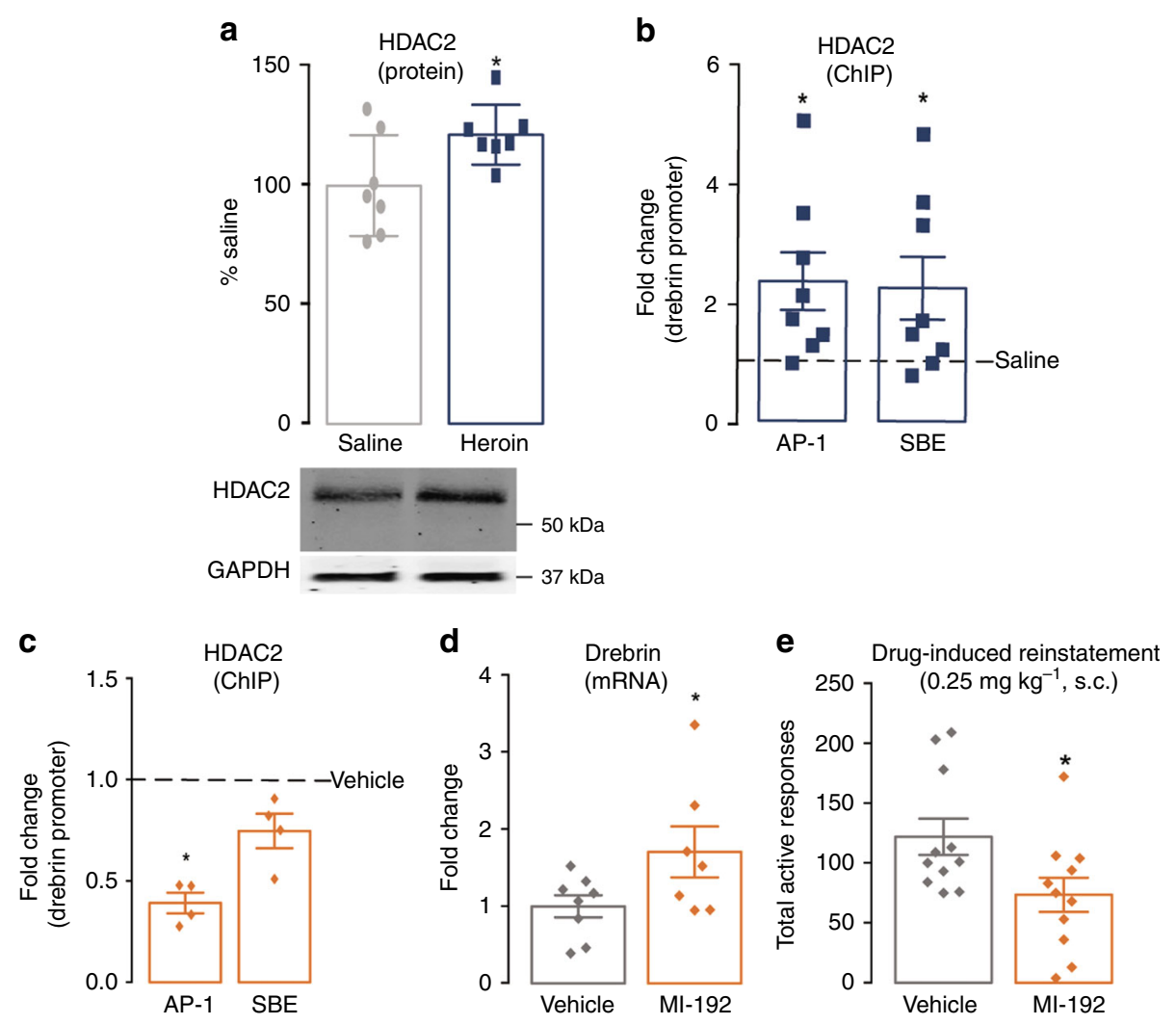

Fig. 2 Epigenetic regulation of drebrin expression following heroin SA. HDAC2 protein expression (Student's t-test: $t_{12}=2.279, P<0.05, n=7$ per group) (a) and binding along AP-1 (Student's $t$-test: $t_{14}=2.435, P<0.05, n=8$ per group) and SBE (Student's t-test: $t_{14}=2.17, P<0.05, n=8$ per group) sites along the drebrin promoter (b) following 10 days of heroin SA. HDAC2 binding along AP-1 (Student's $t$-test: $t_{6}=3.383, P<0.05, n=4$ per group) and SBE (Student's $t$-test: $t_{6}=31.313, P=0.23, n=4$ per group) sites on the drebrin promoter $(\mathbf{c})$, drebrin mRNA levels (Student's t-test: $t_{13}=2.056, P<0.05, n=$ 7-8 per group) (d), and total active responses (Student's $t$-test: $t_{20}=2.329, P<0.05, n=11$ per group) (e) following HDAC2 inhibition via MI-192 microinjections. Data are expressed as means \pm SEMs. ${ }^{\star} P<0.05$

critical regulator of the analgesic properties of opiates and is specific for addiction-like behaviors.

Drebrin rescues heroin-induced synaptic deficits in the NAc. As drebrin is an important mediator of structural plasticity, we aimed to determine its role in heroin-induced spine plasticity. The decrease in spine density on MSNs following heroin SA was reversed by HSV-mediated drebrin overexpression (Fig. 3a, c), particularly through the restoration of thin spines (HSV-GFP, 0.69 spines per $10 \mu \mathrm{M} \pm 0.848$; HSV-drebrin, 2.63 spines per 10 $\mu \mathrm{M} \pm 1.77$; Student's t-test: $\left.t_{16}=2.934, P<0.05\right)$. Conversely, HSV-CRISPR-drebrin potentiated heroin-induced decreases in spine density (Fig. 3b, c), an effect mediated via thin spines (HSV-scrambled, 3.15 spines per $10 \mu \mathrm{M} \pm 0.18$; HSV-CRISPRCas9, 2.13 spines per $10 \mu \mathrm{M} \pm 0.17$; Student's t-test: $t_{10}=2.238$, $P<0.05)$. Dendritic spine density was negatively correlated with total active responses during reinstatement with both drebrin overexpression and drebrin knockdown (Supplementary Fig. $8 \mathrm{a}, \mathrm{b})$, suggesting that the modulation of spines mediates reinstatement behaviors.

Actin cycling governs dendritic spine formation, structure, and maintenance ${ }^{41}$. We therefore examined whether drebrin overexpression was sufficient to normalize opiate-induced decreases in actin stability. Using repeated morphine exposure, which allowed for a temporal regulation of both viral expression and opiate exposure, we showed that F/G-actin ratios were restored by HSV-drebrin (Fig. 4a), which occurred by reversing the opiateinduced decrease in F-actin pools (Supplementary Fig. 9a, b). These actin dynamic-driven alterations in spine density likely altered postsynaptic glutamatergic signaling, which is in part governed by cytoskeletal proteins ${ }^{42}$. Consistent with the loss of dendritic spines induced by heroin, we observed decreases in the surface expression of AMPA and NMDA receptor subunits after SA (Fig. 4b-d). Electrophysiological analyses confirmed that heroin SA decreased both AMPA (Supplementary Fig. 10c) and NMDA (Supplementary Fig. 10d) receptor excitatory postsynaptic currents but did not alter AMPA/NMDA current ratios (Supplementary Fig. 10a; representative traces Supplementary Fig. 10b). Importantly, virus-mediated drebrin expression, which rescued heroin-induced spine density loss, also restored AMPA and NMDA receptor currents (Supplementary Fig. 10c, d).

Opiate-induced plasticity in D1-MSNs. As D1 and D2 MSNs have selective and often opposing roles in responses to drugs of abuse $^{16}$, we next examined whether the expression of drebrin in the NAc and drebrin-mediated plasticity following exposure to opiates was cell-type specific. Using validated transgenic RiboTag technology, we found that following heroin SA (Fig. 5a) in D1and D2-cre transgenic rats ${ }^{43}$ (Supplementary Fig. 11) or repeated administration of morphine (Supplementary Fig. 12) in D1- and D2-ribotag mice, drebrin expression decreased exclusively in D1type MSNs in the NAc. These data also demonstrate that celltype-specific regulation of drebrin is conserved across species (mice, rats), opiate drug (morphine, heroin), and paradigm of drug administration (nonvolitional, volitional). In agreement with our whole-cell findings demonstrating that HDAC2 regulates drebrin expression, we found that HDAC2 was upregulated in D1 but not D2 MSNs (Supplementary Fig. 13) following heroin SA. 
$\mathbf{a}$

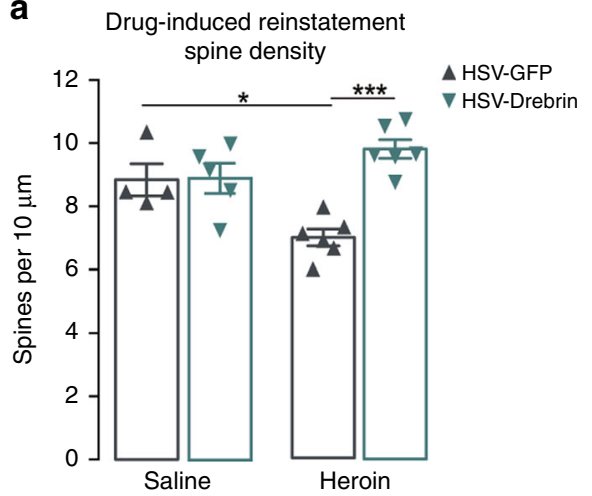

C

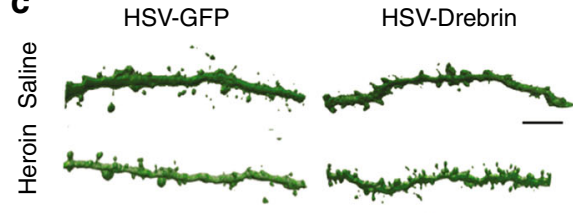

b

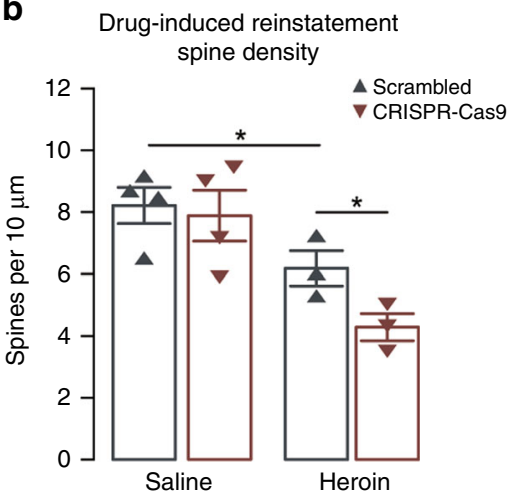

HSV-Scrambled HSV-CRISPR-Cas9

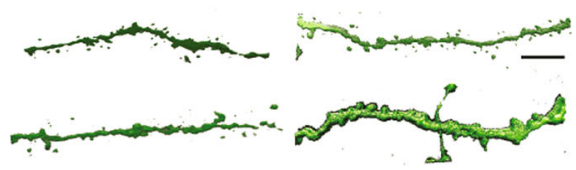

Fig. 3 Drebrin in the NAc regulates opiate-induced structural plasticity. a Spine densities on MSNs in the NAc following intra-NAc HSV-drebrin expression (two-way ANOVA: drug effect, $F_{2,15}=0.482, P<0.05$; virus effect, $F_{1,15}=13.71, P>0.05$; interaction, $F_{2,15}=5.91, P<0.01$, saline-GFP vs heroin-GFP, $P=$ 0.008 ; heroin-GFP vs heroin-drebrin, $P=0.0002$; saline-GFP vs heroin-drebrin, $P=0.125 ; n=5-6$ per group). b Spine densities on MSNs in the NAc following intra-NAC CRISPR-Cas9 drebrin (two-way ANOVA: drug effect, $F_{1,10}=20.92, P<0.001$; virus effect, $F_{1,10}=3.858, P>0.05$; interaction, $F_{1,10}=$ 2.138, $P>0.05$; saline-scrambled vs heroin-scrambled, $P=0.05$; heroin-scrambled vs heroin-CRISPR, $P=0.04 ; n=3-4$ per group). c Representative images of virus-infected neurons; scale bars, $5 \mu \mathrm{m}$. Data are expressed as means \pm SEMs. ${ }^{\star} P<0.05,{ }^{\star \star \star} P<0.001$

a

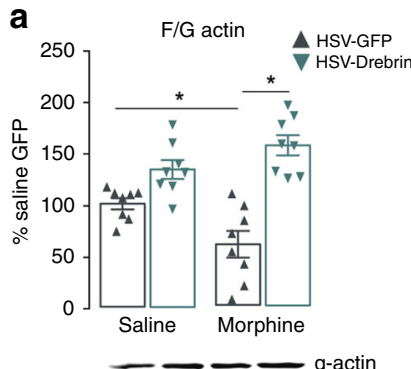

$37 \mathrm{kDa}-$ - - -actin

$37 \mathrm{kDa}_{-}--\frac{\mathrm{f}-\mathrm{actin}}{-}$

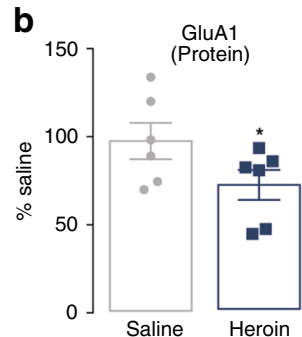

C
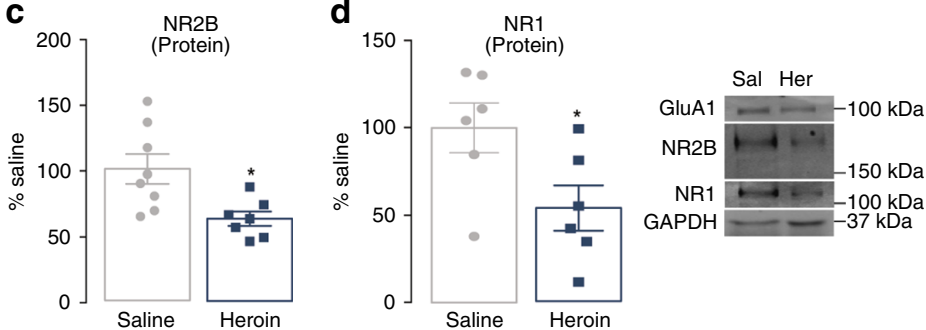

Fig. 4 Drebrin restores opiate-induced deficits in synaptic plasticity. a Intra-NAc expression of HSV-drebrin rescues morphine-induced changes in F/Gactin ratios (two-way ANOVA: drug effect, $F_{1,28}=0.979, P>0.05$; virus effect, $F_{1,28}=45.14, P<0.01$; interaction, $F_{1,28}=10.62, P<0.01$; saline-GFP vs morphine-GFP, $P=0.005$; morphine-GFP vs morphine-drebrin, $P<0.0001$; saline-GFP vs morphine-drebrin, $P=0.0004$; saline-GFP vs saline-drebrin, $P=$ $0.02 ; n=8$ per group). Surface protein expression of AMPA receptor subunit GluA1 (Student's t-test: $t_{10}=1.949, P<0.05, n=6$ per group) (b) and NMDA receptor subunits NR2B (Student's t-test: $t_{13}=2.856, P<0.05, n=7-8$ per group) (c) and NR1 (Student's t-test: $t_{10}=2.405, P<0.05, n=6$ per group) (d) in the NAc following heroin SA. Data are expressed as means \pm SEMs. ${ }^{\star} P<0.05$

To determine if the cell-type-specific decreases in drebrin expression extend to heroin-induced behavioral and structural plasticity, we utilized a Cre-inducible viral vector to overexpress drebrin in Cre-expressing D1 or D2 MSNs. Drebrin overexpression in D1 but not D2 MSNs attenuated drug-induced reinstatement (Fig. 5b, c) and heroin-induced decreases in dendritic spine density (Fig. $5 \mathrm{~d}-\mathrm{f}$ ), with a restoration of thin spines (HSV-GFP, 4.033 spines per $10 \mu \mathrm{M} \pm 0.36$; HSV-LS1L-drebrin, 5.34 spines per $10 \mu \mathrm{M} \pm 0.51$; Student's t-test: $t_{9}=2.152, P<$ 0.05). These data are among the first to highlight a molecular basis for cell-type-specific plasticity following heroin abuse.

\section{Discussion}

We demonstrated that the actin-binding protein drebrin is downregulated in the NAc following heroin administration. Furthermore, we found that drebrin is transcriptionally repressed by the histone modifier HDAC2, an effect that was reversed by pharmacological inhibition of HDACs. Importantly, we demonstrated that the downregulation of drebrin occurs exclusively in D1 MSNs and that restoration of drebrin levels in these cells relieves opiate-induced structural changes and diminishes druginduced craving (Supplementary Fig. 14). Taken together, the results from this study provide the first evidence of a detailed cellular mechanism of relapse to opiates in a cell-type- and circuit-specific manner.

Alterations in synaptic connectivity are a hallmark of many psychiatric disease states, including opioid use disorder ${ }^{19,42,44}$. The excitatory synaptic connections on dendritic spines, including those in the NAc, are modulated by actin cycling ${ }^{45}$. In this study, we found that heroin SA disrupts this finely tuned process, resulting in changes to dendritic spine structure and function in the NAc shell. The observed decrease in glutamatergic tone, 
through both AMPA and NMDA receptor surface expression and currents, is consistent with actin's role as the main anchoring site for these and other postsynaptic proteins ${ }^{46}$. Our data further

a

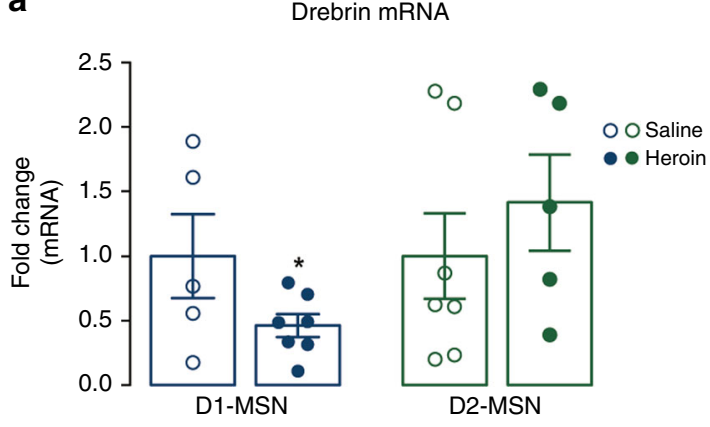

b

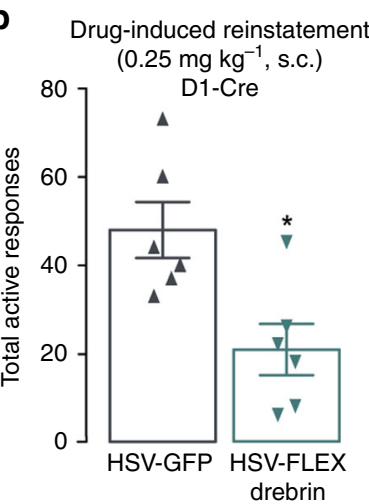

C
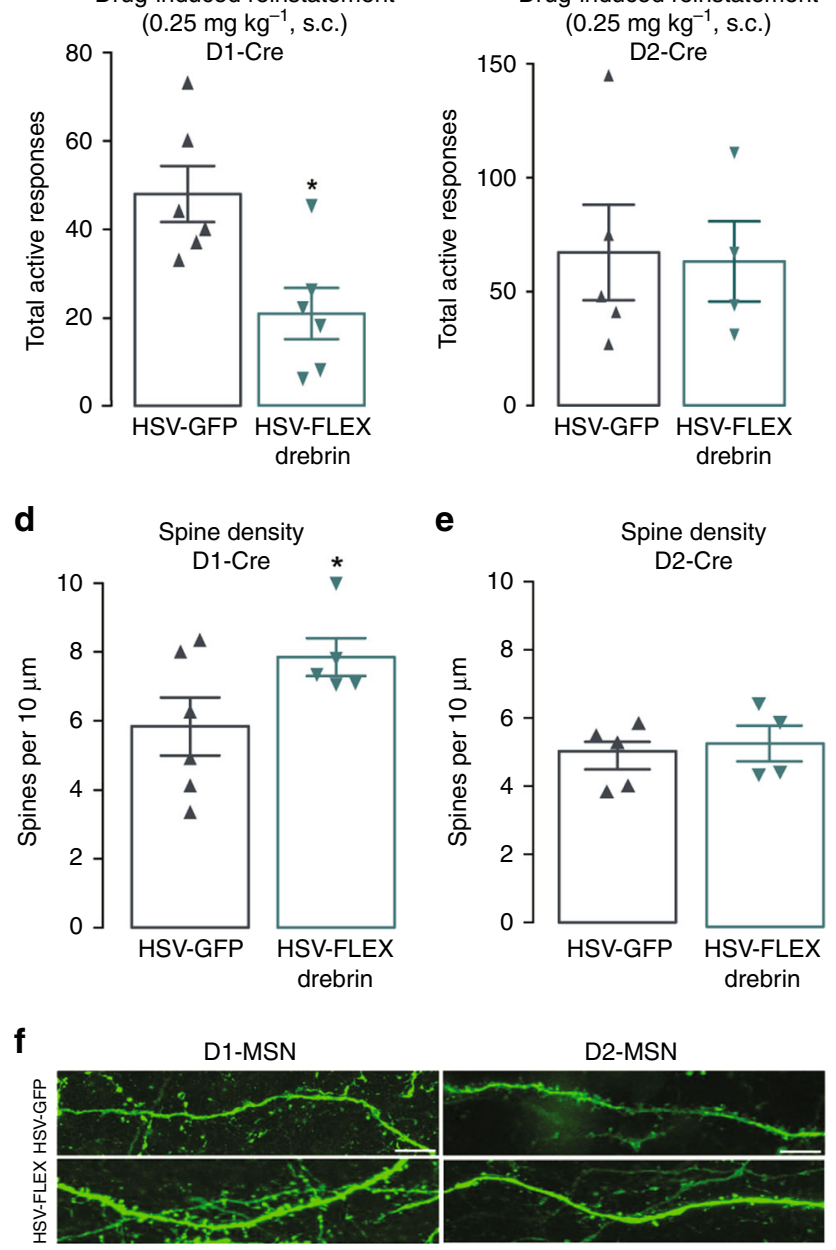

Fig. 5 Cell-type-specific expression of drebrin alters behavioral and structural plasticity. a Drebrin mRNA expression in D1-expressing (Student's t-test: $t_{10}=1.864, P<0.05, n=5-7$ per group) and D2expressing (Student's $t$-test: $t_{10}=0.82, P>0.05, n=5-7$ per group) MSNs following heroin SA. Total active responses following cell-type-specific overexpression of drebrin in D1-expressing (Student's t-test: $t_{10}=3.153$, $P<0.05, n=6$ per group) (b) or D2-expressing (Student's $t$-test: $t_{7}=$ $0.1394, P>0.05, n=4-5$ per group) (c) MSNs. Dendritic spine densities following cell-type-specific overexpression of drebrin in D1-expressing (Student's $t$-test: $t_{9}=1.914, P<0.05, n=5-6$ per group) (d) or D2expressing (Student's $t$-test: $t_{7}=0.537, P>0.05, n=4-5$ per group) (e) MSNs. (f) Representative images of virus-infected neurons; scale bars, $5 \mu \mathrm{m}$. Data are expressed as means \pm SEMs. ${ }^{\star} P<0.05$ indicate that these changes are mediated by drebrin, which binds to F-actin to slow the depolymerization of filaments, the core element of dendritic spines critical for synapse stability and function ${ }^{47}$. Indeed, drebrin-mediated synapse loss is at the center of other pathological disease states marked by spine loss ${ }^{28,29}$, such as Alzheimer's disease, for which the marked decrease in drebrin expression has become a hallmark ${ }^{48}$.

Chromatin remodeling is a central mechanism for the regulation of gene transcription in disorders involving synaptic plasticity, and we demonstrate that this occurs following heroin SA, leading to changes in acetylation along the drebrin promoter. Specifically, we observed an increase in the transcriptional repressor HDAC2 and a concomitant decrease in acetylation of the drebrin promoter. These data are in line with studies which note that HDAC2-knockout mice have a higher spine density in the hippocampus than wild types and that HDAC2 overexpression decreased spine density ${ }^{34}$. We further showed that HDAC inhibition reduced HDAC2 binding along the drebrin promoter, leading to enhanced gene transcription, consistent with studies in which HDAC inhibition restored drebrin levels in a mouse model of Alzheimer's ${ }^{49}$. Most importantly, we showed that HDAC inhibition decreased druginduced reinstatement, providing the first evidence for HDAC2mediated loss of drebrin in the pathophysiology of opiate abuse. While we chose an inhibitor (MI-192) that blocks the function of class I enzymes, HDAC2 and HDAC3, MI-192 exhibits higher selectivity for HDAC2. Future, our data are in agreement with previous preclinical studies of Alzheimer's have identified that overexpression of HDAC2, but not HDAC1 or 3, induces dendritic spine abnormalities ${ }^{50}$, suggesting HDAC2 as a predominant contributor to synaptic plasticity in class I HDAC enzymes.

Following exposure to opiates, there is synaptic rewiring within the NAc, including a decrease in dendritic spine density on MSNs ${ }^{44,20,42,51}$; but see $^{52,53}$, which persists during prolonged abstinence ${ }^{11,44}$. The weakening of dendritic spines, which is observed through immediate changes in immature, thin spines, leads to a persistent decrease in spine density following prolonged withdrawal ${ }^{11}$. We expand on these findings by demonstrating that these alterations are exacerbated by drebrin knockdown and normalized by drebrin overexpression, which in turn govern actin cycling and relapse-like behavior. Further, consistent with the previous literature, which identified changes in glutamatergic tone as a mechanism of dendritic spine retraction ${ }^{11}$, we also note changes in glutamate receptor expression and function. Importantly, the bidirectionality of drebrin expression to mediate both behavioral and neuronal structural plasticity highlights the essential role of drebrin in synaptic integrity associated with the pathophysiology of addiction. Given that the NAc receives inputs from a variety of regions, including the prefrontal cortex, amygdala, and hippocampus, future studies will investigate the input specificity of altered connectivity to these neurons. Recent studies have demonstrated that the D2 MSNs influence silent excitatory synapses and presynaptic neurotransmitter release $\mathrm{e}^{10,11}$, whereas single-cell drop-sequencing studies indicate that acute morphine treatment engages D1 $\mathrm{MSNs}^{54}$, which leads to enhanced morphine reward 9 . Here we find that heroin SA altered drebrin regulation and spine plasticity exclusively in D1 MSNs. Moreover, D1-specific restoration of drebrin levels reversed the effects on dendritic spine density following drug-primed reinstatement, further supporting the role of D1 MSN activation in drug seeking ${ }^{55}$. While our whole-cell findings note changes in dendritic spine changes and glutamatergic tone, our cell-type specific data cannot rule out the possibility that synaptic pruning occurs in D1-MSNs through loss of drebrin, which leads to increased synaptic strengthening on a per spine basis.

These data provide substantial evidence that heroin induces transcriptional repression of drebrin via HDAC2. Furthermore, 
this opiate-induced repression promotes drug-seeking behaviors and structural remodeling in D1 MSNs, which can be reversed via virus-mediated gene therapy and pharmacological HDAC inhibition. Overall, this study identifies a novel direction, both from a transcriptional and cell-type-specific perspective, for therapeutic targeting strategies in the reversal of opiate addiction.

\section{Materials and methods}

Subjects. Subjects included male Sprague-Dawley rats (Envigo Laboratories, Indianapolis, IN). For cell-type specific experiments, transgenic Long-Evans D1iCre (LE-Tg(Drd1a-iCre)3Ottc) and D2-iCre (LE-Tg(Drd2-iCre)1Ottc) rats were obtained from the National Institute on Drug Abuse (NIDA transgenic rat project; RRRC, Columbia, MO) ${ }^{43}$ and crossed with wild type (WT) Long-Evans (Charles River, Wilmington, MA). A breeding colony was maintained for the production of D1-cre and D2-Cre heterozygous male and female rats. Rats were 250-300 grams (males) or 175-200 grams (females) at the beginning of experimental procedures. The homozygous RiboTag (RT) mice on a C57BL/6J background, expressing Creinducible hemagglutinin (HA)-Rpl22 (Jackson Laboratory, Bar Harbor, ME) were crossed with D1-Cre (line FK150) or D2-Cre (line ER44) mouse lines (GENSAT) to generate D1-Cre-RT and D2-Cre-RT mice ${ }^{56}$. Animals were housed at $22-25^{\circ} \mathrm{C}$ under a $12-\mathrm{h} / 12$-h reverse light/dark cycle with lights on at 6:00 pm and ad libitum access to food and water. Testing took place 7 days per week during the dark portion of the light/dark cycle. All animals were maintained according to the National Institutes of Health guidelines in Association for Assessment and Accreditation of Laboratory Animal Care accredited facilities. All experiments and protocols were approved and conducted in accordance with the Institutional Animal Care and Use Committee (IACUC) of The State University of New York at Buffalo, University of Maryland School of Medicine, and California State University at Bakersfield.

Self-administration chambers. The experimental chambers have been described in detail elsewhere ${ }^{32,35,57}$. Briefly, 24 standard Med Associates Inc. chambers (St. Albans, VT), each containing two nose-poke holes with infrared monitoring, were used. Two stimulus lights were mounted above each nose-poke hole, with a house light in the center back wall of the test chamber. All chambers are housed in soundattenuating boxes and controlled through a Med Associates interface.

Drugs. Solutions of heroin hydrochloride and morphine (generously gifted from the NIDA drug supply program), dissolved in $0.9 \%$ sterile saline, were prepared on a weekly basis $\left(0.07 \mathrm{mg} \mathrm{mL}^{-1}\right.$, and $10 \mathrm{mg} \mathrm{mL}^{-1}$, respectively). Pump durations and injection volumes were adjusted according to the animals' body weights on a daily basis to ensure delivery of the correct dose of drug for each animal.

Jugular catheter and patency. Animals were implanted with chronic indwelling jugular catheters, as previously described ${ }^{32,35}$. To preserve patency, catheters were flushed daily with $0.2 \mathrm{~mL}$ of heparinized saline (solution $50 \mathrm{IU} \mathrm{mL}^{-1}$ ) containing enrofloxacin $\left(4 \mathrm{mg} \mathrm{mL}^{-1}\right)$. Patency testing took place once per week throughout the experiments. Catheter patency was tested by injecting ketamine hydrochloride $\left(5 \mathrm{mg} \mathrm{mL}^{-1}\right.$ in $0.5 \mathrm{~mL}$, i.v.) and observing behavioral responses. The loss of the righting reflex and muscle tone were indicators of patency. Only rats with patent catheters were used in data analyses $(<10 \%$ exclusion)

Heroin self-administration. For biochemical experiments, one week after jugular catheter surgery, rats were trained to self-administer heroin $\left(0.02 \mathrm{mg} \mathrm{kg}^{-1}\right.$ infusion -1 [inf]) or saline for $3 \mathrm{~h}$ each day for a total of 10 days (Supplementary Fig. 15a). Responses in the active nose-poke resulted in an infusion of drug, followed by a 20 $s$ time-out period using a fixed ratio (FR) 1 schedule of reinforcement, which was increased daily to an FR3 and maintained at this FR for the remainder of the selfadministration protocol. Following each self-administration session, catheters were flushed, and the rats were returned to the colony room. Tissue collection was performed $24 \mathrm{~h}$ following the last self-administration session. Subjects were rapidly decapitated, and their brains were harvested and sectioned into 1-mm-thick sections from which 1-mm-diameter biopsy punches were taken in regions as previously described ${ }^{58}$.

Sucrose self-administration. Rats were trained to self-administer sucrose (10\%) or water for $3 \mathrm{~h}$ each day for a total of 10 days. Responses in the active nose-poke resulted in the delivery of $88.5 \mu$ l of a sucrose solution directly in the active hole accompanied by a 5-s cue light, followed by a 20 -s time-out period using a fixed ratio (FR) 1 schedule of reinforcement, which was increased daily to FR3 and maintained at this FR for the remainder of the self-administration protocol.

Repeated morphine exposure. Animals were treated with saline or morphine $\left(10 \mathrm{mg} \mathrm{kg}^{-1}\right.$, i.p.) for 10 days. Twenty-four $\mathrm{h}$ following their last injection, tissue punches or whole brains were collected for mRNA or protein analysis.
Locomotor activity. Locomotor activity was recorded via an infrared motionsensor system (AccuScan Instruments, Columbus, $\mathrm{OH}$ ) fitted outside plastic cages $(40 \times 40 \times 30 \mathrm{~cm})$. Plastic cages contained a layer of corn cob bedding and were cleaned between each test session. The Fusion activity-monitoring system software monitors infrared beam breaks at a frequency of $0.01 \mathrm{~s}$, and any interruption of beam that was not interrupted during the previous sample was interpreted as an activity score. The Versa Max animal activity-monitoring software monitored the distance the animal traveled in $1 \mathrm{~h}$.

Viral constructs. The herpes simplex virus (HSV) system allows for expression of proteins exclusively in neuronal cells ${ }^{59}$ that is detectable in vivo for 8 days, with maximal expression occurring on days $3-4^{60}$. Drebrin and scrambled control constructs were obtained from Addgene (Watertown, MA ${ }^{61}$ and cloned into a p1005 backbone under the CMV promoter to produce HSV-drebrin and HSVscrambled. The CRISPER construct was designed by first selecting guide RNAs (gRNAs) against rat drebrin (ENSG00000113758) using the free software at eCRISP.org. The resulting gRNAs were cloned into an all-in-one mammalian expression vector in the configuration hCMV-Cas9-2A-GFP-WPRE-U3 promotergRNA and were tested in vitro for efficiency of drebrin knockdown and specificity. The construct that gave the greatest knockdown (containing the sequence GCCCGCAGCATGGCCGGCGT) was packaged into virus. For cell-type specific behavioral and morphological studies, drebrin was cloned into a cre-dependent transcription cassette (HSV-LS1L-drebrin), which utilizes a lox-stop-lox to confer cre dependence ${ }^{62,63}$. For rat RiboTag studies, recombinant Cre-dependent adenoassociated virus (AAVs) were used in this study. Cre-inducible RiboTag (HARpl22 ${ }^{64}$; gifted from Dr. McKnight) in a EF1a-DIO vector was packaged into AAV (serotype 2$)^{56}$. All viral constructs were validated in vivo.

Stereotaxic surgeries. Rats were counterbalanced on the basis of their behavioral performance (i.e., number of infusions earned during self-administration) and assigned to receive intra-nucleus accumbens (NAc) injections of HSV-drebrin, HSV-GFP, HSV-CRISPR drebrin, HSV-scrambled, or HSV-LS1L-drebrin. The injectors were set at a $10^{\circ}$ angle (measurements on the surface of the skull from bregma taken in $\mathrm{mm}$ : $\mathrm{AP},+1.7$; $\mathrm{ML},+2.45$; $\mathrm{DV},-6.7)$, and the viruses were manually infused at a rate of $0.2 \mu \mathrm{lmin}-1$ for a total of $1.0 \mu \mathrm{l}$ hemisphere ${ }^{-1}$. The injectors were left in place for an additional $10 \mathrm{~min}$ to allow for diffusion. Behavioral and biochemical assays were performed during maximal viral expression (4 days following surgery) with the exception of those with HSV-CRISPR drebrin, which were performed 1 week following maximal expression to allow for optimal protein turnover and degradation (see Supplementary Fig. 4c-e).

Drug- and sucrose-induced reinstatement. Animals were trained to selfadminister heroin $\left(0.02 \mathrm{mg} \mathrm{kg}^{-1} \mathrm{inf}^{-1}\right)$, saline, sucrose $(10 \%)$, or water for 10 days (Supplementary Fig. 15b). Following self-administration (day 11), rats (Sprauge Dawley for HSV-drebrin and HSV-CRISPR experiments and D1-/D2-Cre Long Evans for HSV-LS1L-drebrin experiments) were returned to the experimental chambers for a within-session extinction procedure in which heroin or sucrose administration was withheld, as previously described ${ }^{32,65}$. Briefly, rats were exposed to extinction sessions in the presence of the house light and cues that designated drug/sucrose availability during training sessions. The animals were allowed to respond for 8-10 1-h sessions, separated by 5-min intervals during which the house light was extinguished, until their responses fell to less than 30 responses per session. Following extinction, on day 12, rats were counterbalanced and received viral injections (HSV-drebrin, HSV-GFP, HSV-CRISPR drebrin, HSV-scrambled, or HSV-LS1L-drebrin) in the NAc. Following recovery, when the virus is maximally expressed, rats were then injected with $0.25 \mathrm{mg} \mathrm{kg}^{-1}$ of heroin (s.c.) or primed with $0.2 \mathrm{~mL}$ of sucrose directly in the active hole, returned to the operant chambers, and tested for drug or sucrose reinstatement responding, respectively.

Cannula implantation and HDAC2 inhibition. For the study of HDAC2 inhibition, rats simultaneously underwent jugular catheter surgery and implantation with a bilateral guide cannula (C235F-2.4; Plastics One, Roanoke, VA), aimed at the NAc shell (all coordinates from bregma at the surface of the skull: AP, +1.8; ML, +1.2 ; DV,$-6.5 \mathrm{~mm}$ ). Animals were handled daily and sham injected during the recovery period in order to habituate them to the microinjection procedure. Following 10 days of heroin self-administration $\left(0.02 \mathrm{mg} \mathrm{kg}^{-1} \mathrm{inf}^{-1}\right.$; described above) and extinction (described above in Drug- and sucrose-induced reinstatement; Supplementary Fig. 15c), animals were counterbalanced according to selfadministration performance and assinged to receive microinjections of vehicle or HDAC inhibitor, MI192 (5647; Tocris Bio-Techne, Minneapolis, MN; $1 \mathrm{mM}$ dissolved in a mixture of dimethyl sulfoxide and phosphate-buffered saline [PBS; 1:20; $\left.\mathrm{vol} \mathrm{vol}^{-1}\right]$ and then diluted 1:1,000 in PBS for a final concentration of $1 \mu \mathrm{M}$ ) for a total of $1.0 \mu \mathrm{L}$ hemisphere ${ }^{-1}$. Microinjections were infused at a rate of $0.5 \mu \mathrm{L}$ min ${ }^{-1}$ and injectiors were left in place for $10 \mathrm{~min}$ to allow for diffusion. Microinjections occurred for 3 days, prior to drug-induced reinstatement. Twenty-four $h$ following the last microinjections, rats were placed in operant chambers and tested for drug reinstatement responding as previously described. 
Dose response. Rats were first trained to self-administer heroin for 5 days as described above ( 5 days; $2 \mathrm{~h} \mathrm{day}^{-1} ; 0.02 \mathrm{mg} \mathrm{kg}^{-1} \mathrm{inf}^{-1}$ of heroin; Supplementary Fig. 15d). On day 6, animals were subsequently trained on a within-session doseresponse procedure, as previously described ${ }^{32}$ with slight modifications. Briefly, the 2 -h self-administration session was divided into four 30 -min sessions, each proceeded by a 2-min time-out period. Rats were exposed to four doses of heroin $\left(0.006,0.023,0.045\right.$, and $\left.0.02 \mathrm{mg} \mathrm{kg}^{-1} \mathrm{inf}^{-1}\right)$ for $30 \mathrm{~min}$. The order for the doses tested was pseudorandomized such that the same doses were never tested in the same order during training. We limited each of the four self-administration sessions to $30 \mathrm{~min}$ primarily to match the session duration during training ( $2 \mathrm{~h}$ ) and to delineate drug intake at multiple doses of drug while minimizing drug side effects (i.e., sedation, stereotypy, etc.), which may be a confounding variable. The dose of heroin per injection was regulated via adjusting the infusion volumes and pump durations. Following each test session, the catheters were flushed and rats were returned to the colony room. Following the 5 days of dose-response training (on day 11), rats received virus infections (HSV-GFP, HSV-drebrin, HSV-scrambled, or HSV-CRISPR drebrin) in the NAc. Following recovery, when the virus is maximally expressed, animals were placed back in the operant chambers and retested on the within-session dose-response procedure described above.

Dendritic spine analysis. Rats injected with HSV constructs were killed $4 \mathrm{~h}$ after heroin-induced reinstatement tests via transcardial perfusion of PBS $(0.9 \%$ saline $\left[\mathrm{wt} \mathrm{vol}^{-1}\right]$ ) followed by a $4 \%$ formaldehyde (wt vol ${ }^{-1}$ ) solution. The brains were immersed in fixative overnight and then stored in PBS with $0.01 \%$ sodium azide $\left(\mathrm{wt} \mathrm{vol}^{-1}\right.$ ). The brains were sectioned at $100 \mu \mathrm{m}$ on a Vibratome (VT1000S; Leica Microsystems, Wetzlar, Germany) and blocked in 3\% normal donkey serum (wt $\mathrm{vol}^{-1}$ ) with $0.3 \%$ Triton $\mathrm{X}-100\left(\mathrm{vol} \mathrm{vol}^{-1}\right)$ for $2 \mathrm{~h}$ at $4{ }^{\circ} \mathrm{C}$. Sections were then incubated overnight at $22-25^{\circ} \mathrm{C}$ in primary antibody $(1: 1,000$ anti-rabbit GFP; Molecular Probes of Thermo Fisher Scientific, Waltham, MA) diluted in PBS with $3 \%$ normal donkey serum and $0.3 \%$ Tween 20 . Tissue sections were rinsed and incubated overnight at $4{ }^{\circ} \mathrm{C}$ in anti-rabbit secondary antibody $(1: 1,000$; Jackson ImmunoResearch Laboratories, West Grove, PA). Immunofluorescence was imaged on an LSM 510 Meta confocal microscope (Carl Zeiss, Oberkochen, Germany) with a $63 \times$ oil-immersion lens objective. Images were acquired with a pinhole set at 1 arbitrary unit and a 1,024 × 1,024 frame size. Dendritic length was measured using ImageJ software, and spine numbers were counted. The average number of spines per $10 \mu \mathrm{m}$ of dendrite was calculated. An average was obtained from 6-10 neurons per rat ( $n=3-6$ for each of four groups). Medium spiny neurons (MSNs) were located in the NAc shell. Only secondary dendrites that were at least $75 \mu \mathrm{m}$ from the cell body and that were able to be traced back to the cell body were selected for analysis. Spine type analysis was carried out as previously described $^{32}$. Briefly, Neuron Studio (http://research.mssm.edu/cnic/tools-ns.html) was used to analyze dendritic length, width, spine number, and spine head diameter in three dimensions which allowed for classification into major morphological subtypes correlated with spine structure and function. Investigators were blind to the experimental conditions conducted for all confocal acquisitions and analyses of spines.

Induction of inflammatory pain. After rats received virus infections in the NAc, inflammatory pain was induced by injecting the foot pads of the right hind paws with $0.1 \mathrm{~mL}$ complete Freund's adjuvant (Thermo Fisher, Waltham, MA), which contains $0.05 \mathrm{mg}$ of Mycobacterium butyricum dissolved in paraffin oil.

Place escape/avoidance paradigm. The place escape/avoidance paradigm, a model of the affective component of pain, was measured as previously described ${ }^{66}$. To examine the effects virus-mediated gene expression had on drug-induced escape/avoidance learning behaviors, morphine $\left(10 \mathrm{mg} \mathrm{kg}^{-1}\right.$, i.p.) was administered $30 \mathrm{~min}$ before the behavioral testing session. Briefly, rats were placed in inverted cages, painted half white and half black. The cages were placed on an elevated metal grid to enable the visualization and stimulation of the plantar surface of the hind paw with a 60 -g von Frey hair every $15 \mathrm{~s}$ over a 30 -min session. When the rat was on the white side of the chamber, the noninflamed (left) paw was mechanically stimulated; while on the black side, the inflamed paw (right) was mechanically stimulated. The location of the rat in the chamber at the time of stimulation was recorded. The percentage of the time spent on the white side of the chamber was calculated every $15 \mathrm{~s}$ during each 5 -min period and was used as an indication of escape/avoidance learning.

Mechanical hyperalgesia and tolerance induction. Mechanical hyperalgesia was assessed as previously described ${ }^{67}$ with minor modifications. For this experiment, animals were treated with saline and then cumulative doses of morphine $(1.78,3.2$, 5.6 , or $10 \mathrm{mg} \mathrm{kg}^{-1}$ [i.p.]). Hyperalgesia was measured using calibrated von Frey filaments (1.4-26 g; North Coast Medical, Morgan Hill, CA). The rats were placed in elevated plastic chambers on a mesh wire floor. Von Frey filaments were applied to the medial plantar surface of the hind paw in ascending order until buckling occurred and maintained for approximately $2 \mathrm{~s}$. The mechanical threshold (expressed as percent maximal possible effect [\%MPE]) corresponds to the lowest filament force that elicited a behavioral response (paw withdrawal) in at least two of three applications of the filament. The tests were performed in cumulative dosed multiple-cycle procedures, with measurements taken immediately prior to and $20 \mathrm{~min}$ after drug administration before the next drug dose was delivered. These cycles continued until an MPE near $100 \%$ was reached (26 g) or until a dose caused generalized behavioral suppression. Forces larger than $26 \mathrm{~g}$ would physically elevate a non-complete Freund's adjuvant-treated paw and therefore are not reflective of pain-like behavior. Immediately following the von Frey testing, tolerance was induced in animals over 3 days as previously described ${ }^{68}$ with minor modifications. Briefly, rats were treated twice daily with escalating doses of morphine $(20,40,60$ 80 , and $100 \mathrm{mg} \mathrm{kg}^{-1}$ ) over 3 days. Twenty-four h after the last dose, the rats were tested for mechanical hyperalgesia as described above with saline and cumulative doses of morphine $\left(17.8,32,56\right.$, and $100 \mathrm{mg} \mathrm{kg}^{-1}$, [i.p.]).

Western blot analysis. NAc tissue punches from each rat that self-administered heroin or saline were homogenized in $25 \mathrm{mM}$ Tris $(\mathrm{pH} \mathrm{8.0)}$ and $0.25 \mathrm{M}$ sucrose buffer with phosphatase inhibitor cocktails 2 and 3 (Sigma-Aldrich, St. Louis, MO) and protease inhibitors (Roche, Basel, Switzerland). Samples were centrifuged at $21,000 \mathrm{rpm}$ for $10 \mathrm{~min}$ to remove debris. Protein concentrations were determined, and $30 \mu \mathrm{g}$ was loaded onto a $4-15 \%$ gradient Tris-sodium dodecyl sulfate (SDS) gels (Bio-Rad Laboratories, Hercules, CA) for SDS-polyacrylamide gel electrophoresis (PAGE) and then transferred to a BioTrace nitrocellulose membrane (Pall Biotech, Show Low, AZ) and blocked in 5\% nonfat milk. All membranes were incubated overnight at $4{ }^{\circ} \mathrm{C}$. Primary antibodies were diluted in blocking buffer (Rockland Immunochemicals, Inc., Limerick, PA), including those against drebrin (1:500; Cell Signaling Technologies, Inc., Danvers, MA), HDAC2 (1:1000; Cell Signaling Technologies, Inc.), actin (1:500; Cytoskeleton, Inc., Denver, CO), GluA1 (1:100; Santa Cruz Biotechnology, Dallas, TX), NR2B (1:500; Millipore, Darmstadt, Germany), NR1 (1:500; Millipore), and GAPDH (1:10,000; Cell Signaling Technologies, Inc.). Membranes were incubated for $1 \mathrm{~h}$ at room temperature with IRDye secondary antibodies (1:5,000; LI-COR, Inc., Lincoln, NE). The membranes were imaged using the Odyssey infrared imaging system (LI-COR, Inc.) and quantified by densitometry using Image J (National Institute of Health, Bethesda, MD). Full length blots for the main text can be found in Supplementary Fig. 16.

F/G-actin in vivo assay. The ratio of $\mathrm{F}$ actin to $\mathrm{G}$ actin in cells was analyzed using an F-actin/G-actin in vivo assay kit (catalog number BK037; Cytoskeleton Inc.) according to the manufacturer's protocol. Briefly, NAc tissue punches, collected $30-$ min after the last injection, were lysed $\mathrm{w}$ with a motorized pestle homogenizer in a heated $\left(37^{\circ} \mathrm{C}\right)$ solution containing lysis and F-actin stabilization buffer (catalog number LAS01), ATP (catalog number BSA04) and protease inhibitor cocktail (catalog number PIC02). Following a $10 \mathrm{~min}$ incubation at $37^{\circ} \mathrm{C}$ lysates were centrifuged $(350 \mathrm{x} \mathrm{g})$ for $10 \mathrm{~min}$ to pellet debris. Supernatants were collected and centrifuged at $100,000 \times g$ for $60 \mathrm{~min}$ at $37^{\circ} \mathrm{C}$. The supernatants (G-actin) were separated from the pellets (F-actin) and were immediately placed on ice. The pellets (F-actin) were resuspended in F-actin depolymerizing buffer (catalog number FAD02) and incubated on ice for $1 \mathrm{~h}$, pipetting up and down every $15 \mathrm{~min}$. It should be noted differences in resuspension buffers result in different band widths during SDS-PAGE separation. Equal amounts of the samples were loaded in each lane and analyzed by Western blotting with an anti-actin antibody (catalog number AAN01).

Polyribosome immunoprecipitations and RNA isolation. Polyribosomes were immunoprecipitated as previously described ${ }^{56}$. The NAc of D1-Cre rats and D2Cre rats, which received bilateral injections of Cre-inducible RiboTag (HA$\mathrm{Rpl} 22^{64}$ ), were dissected under basal conditions for validation purposes (Supplementary Fig. 11) or after heroin self-administration. The NAc of D1-Cre-RT and D2-Cre-RT mice was dissected following repeated i.p. morphine administration. Four 14-gauge NAc punches were pooled from one or four animals, respectively and homogenized by douncing in a supplemented homogenization buffer $(50 \mathrm{mM}$ Tris, $100 \mathrm{mM} \mathrm{KCl,} 12 \mathrm{mM} \mathrm{MgCl}_{2}, 1 \% \mathrm{NP}-40,1 \mathrm{mM}$ dithiothreitol, $100 \mathrm{ug} \mathrm{mL}^{-1}$ cyclohexamide, $1 \mathrm{mg} \mathrm{mL}^{-1}$ heparin, $200 \mathrm{U} \mathrm{mL}^{-1} \mathrm{RNasin}$, and protein inhibitors) The supernatants $(800 \mu \mathrm{l})$ were added directly to HA-coupled beads and rotated overnight at $4{ }^{\circ} \mathrm{C}$. The beads were then washed three times for $5 \mathrm{~min}$ in a high-salt buffer (50 mM Tris [pH 7.4], $300 \mathrm{mM} \mathrm{KCl}, 12 \mathrm{mM} \mathrm{MgCl}_{2}, 1 \% \mathrm{NP}-40,1 \mathrm{mM}$ dithiothreitol, $100 \mathrm{ug} \mathrm{mL}^{-1}$ cyclohexaminde) on a magnetic rack. Following the washes, RNA was extracted using the TRK lysis buffer provided in the MicroElute total RNA kit (Omega Bio-tek, Inc., Norcross, GA) according to the manufacturer's instructions. RNA was quantified by using a NanoDrop spectrophotometer (ND100; Thermo Fisher Scientific, Hampton, NH). cDNA synthesis and quantitative PCR (qPCR) were carried out as described below.

RNA isolation and qPCR. NAc tissue punches were collected 1 day after the last heroin administration and immediately stored at $-80^{\circ} \mathrm{C}$. RNA was isolated and purified from these samples using Trizol (Invitrogen, Carlsbad, CA) and the MicroElute total RNA kit (Omega Bio-tek, Inc, Norcross, GA) with a DNase step. RNA concentrations were measured on a NanoDrop spectrophotometer, and $500 \mathrm{ng}$ cDNA was then synthesized using an iScript cDNA synthesis kit (Bio-Rad Laboratories, Hercules, CA). mRNA expression changes were measured by RTPCR with IQ SYBR Green Supermix (Bio-Rad Laboratories, Hercules, CA) and quantified using an iQ5 system (Bio-Rad Laboratories, Hercules, CA). Reactions 
were run in triplicates using the $\Delta \Delta C_{T}$ method as described previously using Gapdh as a housekeeping gene. All primer sequences can be found in Table S1.

Chromatin immunoprecipitation (ChIP). ChIP was performed for anti-HDAC2, and anti-acetylated $\mathrm{H} 3$ as previously described ${ }^{12,69}$ with minor modifications. Briefly, four NAc punches from two rats taken $24 \mathrm{~h}$ following the last selfadministration session were pooled for each sample. These punches were immediately fixed in $1 \%$ formaldehyde for $12 \mathrm{~min}$ and then quenched in $2 \mathrm{M}$ glycine for 5 min. Chromatin was sheared using a Biorupter pico 300 (Diagenode Diagnostics, Seraing, Belgium) at $4{ }^{\circ} \mathrm{C}$ at a high sonication intensity for 30 -s on and 30 -s off for $10 \mathrm{~min}$ followed by a rest, which was repeated a total of three times. Chromatin fragment sizes of 250-1,000 bp were verified by agarose gel electrophoresis. Magnetic sheep anti-rabbit beads (Invitrogen, Carlsbad, CA) were incubated with antiHDAC2 (Abcam, Cambridge, MA), anti-acetylated H3 (Millipore, Billerica, MA), and anti-acetylated H4 (Millipore, Billerica, MA) antibodies rotating overnight at $4{ }^{\circ} \mathrm{C}$. After washing, $70 \mu \mathrm{L}$ of the magnetic bead and antibody slurry was incubated with sheared chromatin for $16 \mathrm{~h}$ at $4{ }^{\circ} \mathrm{C} ; 5 \%$ of the sheared chromatin was saved as an input control. The samples were washed with lithium chloride and Trisethylenediaminetetraacetic acid (EDTA) buffer. Reverse cross-linking took place overnight at $65^{\circ} \mathrm{C}$, and proteins and RNA were removed using proteinase $\mathrm{K}$ (Invitrogen, Carlsbad, CA) and RNase (Roche, Basel, Switzerland), respectively. DNA was purified using a DNA purification kit (Qiagen, Hilden, Germany) Immunoglobulin $\mathrm{G}$ was used to control for nonspecific binding. $\mathrm{qPCR}$ was performed to determine if HDAC2, and acetyl H3 were bound to proximal promoter regions of $D b n 1$. Amplification reactions were run in triplicates with iQ SYBR green (Bio-Rad Laboratories, Hercules, CA), and each sample was normalized to an immunoglobulin $\mathrm{G}$ control. Fold changes in expression in animals treated with heroin were calculated relative to that of the saline controls. Primer sequences were tested for efficiency and can be found in Table S2.

Biotinylation assay. Surface AMPA and NDMA receptors were detected as previously described ${ }^{65,70}$. Briefly, bilateral NAc slices were incubated in artificial cerebral spinal fluid containing $1 \mathrm{mg} \mathrm{ml}^{-1} \mathrm{NH}-\mathrm{S}$-S biotin for $30 \mathrm{~min}$ at $4{ }^{\circ} \mathrm{C}$. Glycine was added to the mixture to quench the reaction, washed three times in lysis buffer ( $25 \mathrm{mM}$ HEPES, $500 \mathrm{mM} \mathrm{NaCl}, 2 \mathrm{mM}$ EDTA, $1 \mathrm{mM}$ phenylmethyl sulfonyl fluoride, $20 \mathrm{mM} \mathrm{NaF}, 0.1 \% \mathrm{NP}-40$, protease inhibitor) and homogenized. Cell homogenate was incubated with Neutravidin agarose beads overnight at $4{ }^{\circ} \mathrm{C}$. Following washes, the bound fraction was collected and analyzed by SDS-PAGE as described above.

Electrophysiological recordings. Whole-cell patch-clamp experiments were performed with a Multiclamp $700 \mathrm{~A}$ amplifier and Digdata 1322 A data acquisition system (Molecular Devices, San Jose, CA). Following 10 days of heroin SA, animals were bilaterally injected with HSV-GFP or HSV-drebrin. On day 4, during maximal viral expression, coronal slices $(350 \mu \mathrm{m})$ containing the NAc were prepared using a Vibratome. The slices were placed in a holding chamber containing artificial cerebrospinal fluid ( $130 \mathrm{mM} \mathrm{NaCl}, 26 \mathrm{mM} \mathrm{NaHCO}, 3 \mathrm{mM} \mathrm{KCl}, 5 \mathrm{mM}$ $\mathrm{MgCl}_{2}, 1.25 \mathrm{mM} \mathrm{NaH}_{2} \mathrm{PO}_{4}, 1 \mathrm{mM} \mathrm{CaCl}$, and $10 \mathrm{mM}$ glucose [pH 7.4]; 300 mOsm) oxygenated with $95 \% \mathrm{O}_{2}$ and $5 \% \mathrm{CO}_{2}$ and were maintained at room temperature for at least $1 \mathrm{~h}$. For recording of GFP-labeled NAc MSNs, one slice was positioned in a perfusion chamber attached to the fixed stage of an upright microscope (Olympus, Tokyo, Japan) and submerged in continuously flowing oxygenated artificial cerebrospinal fluid. A bipolar stainless-steel stimulating electrode (FHC, Bowdoin, ME) was placed $100 \mu \mathrm{m}$ rostral to the recording electrode. An S48 stimulator and photoelectric stimulus isolation unit (SIU7) were used to drive the stimulating electrode. The patch electrodes contained an internal solution comprising $130 \mathrm{mM}$ Cs-methanesulfonate, $10 \mathrm{mM} \mathrm{CsCl}, 4 \mathrm{mM} \mathrm{NaCl}, 10 \mathrm{mM}$ HEPES, $1 \mathrm{mM} \mathrm{MgCl}_{2}$, $5 \mathrm{mM}$ EGTA, $2.2 \mathrm{mM}$ QX-314, $12 \mathrm{mM}$ phosphocreatine, $5 \mathrm{mM}$ ATP, $0.5 \mathrm{mM}$ GTP at $\mathrm{pH} 7.2-7.3$ and $270-280 \mathrm{mOsm}$. The resistance of the patch electrodes was $3.0 \mathrm{M} \Omega$. Tight seals $(>2 \mathrm{G} \Omega$ ) were obtained by applying negative pressure. The membranes were disrupted with additional suction, and the whole-cell configuration was obtained. The membrane capacitance of all recorded cells was between 40 and $50 \mathrm{pF}$ and the series resistance was $<20 \mathrm{M} \Omega$. AMPAR and NMDAR-mediated evoked excitatory postsynaptic currents (eEPSCs) and their ratios were obtained in the presence of bicuculline $(10 \mu \mathrm{M})$ at holding potentials of $-70 \mathrm{mV}$ and $+40 \mathrm{mV}$, respectively. The NMDAR-mediated eEPSC was calculated $40 \mathrm{~ms}$ after the stimulus artifact. The stimulation intensity was set at $0.2 \mathrm{~ms}$ and $8 \mathrm{~V}$ for the $\mathrm{S} 48$ stimulator and at the $10-150 \mu \mathrm{A}$ range of isolation unit SIU7. Under this stimulation intensity, the AMPAR-mediated eEPSCs from most of the control cells were between 200 and $400 \mathrm{pA}$. The stimulation intensity setting was kept the same for all eEPSC recordings. Each evoked response was for $3 \mathrm{~min}$ with an interstimulus interval of $20 \mathrm{~s}$ for all the cells measured. Data analyses were performed with Clampfit software (Axon Instruments of Molecular Devices).

Statistical analyses. All statistical analyses were performed using GraphPad Prism (GraphPad Software, San Diego, CA). The primary dependent measures were the number of infusions for self-administration (drug and sucrose), the number of total active responses during drug-induced reinstatement, spine density, fold change (mRNA), relative density (protein), and fold change (ChIP). Shapiro-Wilks tests of normality and Bartlett's tests of homogeneity of variance were conducted to test for normal distributions. In events that a normal distribution could not be assumed, nonparametric tests were used. Performance during self-administration was analyzed using a repeated measures two-factor within-subject analysis of variance (ANOVA), with drug (heroin or saline) as the between-session variable and time (day of testing) as the within-subject variable using Fishers least significant difference (LSD) post hoc tests to determine the source of significance. Performance during the within-session dose response was analyzed using a two-factor ANOVA, with virus (HSV-GFP, HSVdrebrin, HSV-scrambled, or HSV-CRISPR-Cas9) as the between-session variable and dose as the within-subject variable, using Fisher's LSD post hoc tests to determine the source of significance. Performance during drug reinstatement and subsequent spine density changes were analyzed using a two-way ANOVA, with virus (HSV-GFP, HSV-drebrin, HSV-scrambled, or HSV-CRISPR-Cas9) and drug treatment (heroin or saline) as the dependent variables; Fisher's LSD post hoc tests to determine the source of significance. Electrophysiological recordings were analyzed using a one-way ANOVA on ranks with Dunn's post hoc analysis. Student's $t$ tests were conducted on measures of locomotor activity, Western blotting data, and mRNA levels followed by Fisher's LSD post hoc tests. Tests were two tailed except where directional hypotheses could be inferred. Significance was set at a $P$ value of $<0.05$, and data are presented as the means \pm SEMs.

Reporting summary. Further information on research design is available in the Nature Research Reporting Summary linked to this article.

\section{Data availability}

The data that support the findings of this study are available from the corresponding author upon reasonable request.

Received: 7 June 2019 Accepted: 22 August 2019

Published online: 12 September 2019

\section{References}

1. Jones, C. M., Logan, J., Gladden, M. \& Bohm, M. K. Vital signs: demographic and substance use trends among heroin users-United States, 2002-2013. Mmwr. Morb. Mortal. Wkly. Rep. 64, 719-725 (2015).

2. Scholl, L., Karissa, S. P., Wilson, M. \& Baldwin, N. F. Drug and OpioidInvolved Overdose Deaths-United States, 2013-2017. MMWR Morb Mortal Wkly Rep. 67, 1419-1427 (2019).

3. Bhambhvani, H. P., Simmons, M., Haroutunian, V. \& Meador-Woodruff, J. H. Decreased expression of cortactin in the schizophrenia brain. Neuroreport 27, 145-150 (2016).

4. Shankar, G. M. et al. Natural oligomers of the Alzheimer amyloid- $\beta$ protein induce reversible synapse loss by modulating an NMDA-type glutamate receptor-dependent signaling pathway. J. Neurosci. 27, 2866 (2007).

5. Hutsler, J. J. \& Zhang, H. Increased dendritic spine densities on cortical projection neurons in autism spectrum disorders. Brain Res. 1309, 83-94 (2010).

6. Shen, H., Moussawi, K., Zhou, W., Toda, S. \& Kalivas, P. W. Heroin relapse requires long-term potentiation-like plasticity mediated by NMDA2bcontaining receptors. Proc. Natl Acad. Sci. USA 108, 19407-19412 (2011).

7. LaLumiere, R. T. \& Kalivas, P. W. Glutamate release in the nucleus accumbens core is necessary for heroin seeking. J. Neurosci. 28, 3170-3177 (2008).

8. Robison, A. J. \& Nestler, E. J. Transcriptional and epigenetic mechanisms of addiction. Nat. Rev. Neurosci. 12, 623-637 (2011).

9. Koo, J. W. et al. Loss of BDNF signaling in D1R-expressing NAc neurons enhances morphine reward by reducing GABA inhibition. Neuropsychopharmacology 39, 2646-2653 (2014).

10. Hearing, M. C. et al. Reversal of morphine-induced cell-type-specific synaptic plasticity in the nucleus accumbens shell blocks reinstatement. Proc. Natl Acad. Sci. USA 113, 757-762 (2016).

11. Graziane, N. M. et al. Opposing mechanisms mediate morphine- and cocaineinduced generation of silent synapses. Nat. Neurosci. 19, 915-925 (2016).

12. Lobo, M. K. et al. Cell type-specific loss of BDNF signaling mimics optogenetic control of cocaine reward. Science.330, 385-390 (2010).

13. Chandra, R. et al. Opposing role for Egr3 in nucleus accumbens cell subtypes in cocaine action. J. Neurosci. 35, 7927-7937 (2015).

14. Kupchik, Y. M. et al. Coding the direct/indirect pathways by D1 and D2 receptors is not valid for accumbens projections. Nat. Neurosci. 18, $1230-+(2015)$

15. Lobo, M. K., Karsten, S. L., Gray, M., Geschwind, D. H. \& Yang, X. W. FACSarray profiling of striatal projection neuron subtypes in juvenile and adult mouse brains. Nat. Neurosci. 9, 443-452 (2006).

16. Lobo, M. K. \& Nestler, E. J. The striatal balancing act in drug addiction: distinct roles of direct and indirect pathway medium spiny neurons. Front. Neuroanat., https://doi.org/10.3389/fnana.2011.00041 (2011). 
17. Gerfen, C. R. The neostriatal mosaic: multiple levels of compartmental organization. Trends Neurosci. 15, 133-139 (1992).

18. Spiga, S., Puddu, M. C., Pisano, M. \& Diana, M. Morphine withdrawalinduced morphological changes in the nucleus accumbens. Eur. J. Neurosci. 22, 2332-2340 (2005).

19. Robinson, T. E., Gorny, G., Savage, V. R. \& Kolb, B. Widespread but regionally specific effects of experimenter- versus self-administered morphine on dendritic spines in the nucleus accumbens, hippocampus, and neocortex of adult rats. Synapse 46, 271-279 (2002).

20. Robinson, T. E. \& Kolb, B. Morphine alters the structure of neurons in the nucleus accumbens and neocortex of rats. Synapse 33, 160-162 (1999).

21. Wang, Z., Yan, P., Hui, T. \& Zhang, J. Epigenetic upregulation of PSD-95 contributes to the rewarding behavior by morphine conditioning. Eur. J. Pharmacol. 732, 123-129 (2014).

22. Golden, S. A. et al. Epigenetic regulation of RAC1 induces synaptic remodeling in stress disorders and depression. Nat. Med. 19, 337-344 (2013).

23. Jung, G. et al. Drebrin depletion alters neurotransmitter receptor levels in protein complexes, dendritic spine morphogenesis and memory-related synaptic plasticity in the mouse hippocampus. J. Neurochem. 134, 327-339 (2015).

24. Kojima, N. \& Shirao, T. Synaptic dysfunction and disruption of postsynaptic drebrin-actin complex: a study of neurological disorders accompanied by cognitive deficits. Neurosci. Res. 58, 1-5 (2007).

25. Renthal, W. \& Nestler, E. J. Epigenetic mechanisms in drug addiction. Trends Mol. Med. 14, 341-350 (2008).

26. Covington, H. E. III et al. Antidepressant actions of histone deacetylase inhibitors. J. Neurosci. 29, 11451-11460 (2009).

27. Whittle, N. \& Singewald, N. HDAC inhibitors as cognitive enhancers in fear, anxiety and trauma therapy: where do we stand? Biochem. Soc. Trans. $\mathbf{4 2}$, 569-581 (2014).

28. Dun, X.-p \& Chilton, J. K. Control of cell shape and plasticity during development and disease by the actin-binding protein Drebrin. Histol. Histopathol. 25, 533-540 (2010).

29. Hill, J. J., Hashimoto, T. \& Lewis, D. A. Molecular mechanisms contributing to dendritic spine alterations in the prefrontal cortex of subjects with schizophrenia. Mol. Psychiatry 11, 557-566 (2006).

30. Childress, A. R. et al. Cue reactivity and cue reactivity interventions in drug dependence. NIDA Res. Monogr. 137, 73-95 (1993).

31. Piazza, P. V., Deroche-Gamonent, V., Rouge-Pont, F. \& Le Moal, M. Vertical shifts in self-administration dose-response functions predict a drug-vulnerable phenotype predisposed to addiction. J. Neurosci. 20, 4226-4232 (2000).

32. Gancarz, A. M. et al. Activin receptor signaling regulates cocaine-primed behavioral and morphological plasticity. Nat. Neurosci. https://doi.org/ 10.1038/nn.4036 (2015).

33. Renthal, W. \& Nestler, E. J. Histone acetylation in drug addiction. Semin. Cell. Dev. Biol. 20, 387-394 (2009).

34. Guan, J. S. et al. HDAC2 negatively regulates memory formation and synaptic plasticity. Nature 459, 55-U58 (2009).

35. Wang, Z.-J. et al. BRG1 in the nucleus accumbens regulates cocaine-seeking behavior. Biol. Psychiatry 80, 652-660 (2016).

36. Durham, B. S., Grigg, R. \& Wood, I. C. Inhibition of histone deacetylase 1 or 2 reduces induced cytokine expression in microglia through a protein synthesis independent mechanism. J. Neurochem. 143, 214-224 (2017).

37. Dumas, E. O. \& Pollack, G. M. Opioid tolerance development: a pharmacokinetic/pharmacodynamic perspective. Aaps. J. 10, 537-551 (2008).

38. Descalzi, G. et al. Epigenetic mechanisms of chronic pain. Trends Neurosci. 38, 237-246 (2015).

39. Bushnell, M. C., Ceko, M. \& Low, L. A. Cognitive and emotional control of pain and its disruption in chronic pain. Nat. Rev. Neurosci. 14, 502-511 (2013).

40. Price, D. D. Neuroscience-Psychological and neural mechanisms of the affective dimension of pain. Science 288, 1769-1772 (2000).

41. Spence, E. F. \& Soderling, S. H. Actin out: regulation of the scnaptic Cytoskeleton. J. Biol. Chem. 290, 28613-28622 (2015).

42. Russo, S. J. et al. The addicted synapse: mechanisms of synaptic and structural plasticity in nucleus accumbens. Trends Neurosci. 33, 267-276 (2010).

43. Pardo-Garcia, T. R. et al. Ventral pallidum is the primary target for accumbens D1 projections driving cocaine seeking. J. Neurosci. 39, 2041-2051 (2019).

44. Robinson, T. E. \& Kolb, B. Structural plasticity associated with exposure to drugs of abuse. Neuropharmacology 47, 33-46 (2004).

45. Toda, S., Shen, H. \& Kalivas, P. W. Inhibition of actin polymerization prevents cocaine-induced changes in spine morphology in the nucleus accumbens. Neurotox. Res. 18, 410-415 (2010).

46. Allison, D. W., Gelfand, V. I., Spector, I. \& Craig, A. M. Role of actin in anchoring postsynaptic receptors in cultured hippocampal neurons: differential attachment of NMDA versus AMPA receptors. J. Neurosci. 18, 2423-2436 (1998).

47. Ivanov, A., Esclapez, M., Pellegrino, C., Shirao, T. \& Ferhat, L. Drebrin A regulates dendritic spine plasticity and synaptic function in mature cultured hippocampal neurons. J. Cell. Sci. 122, 524-534 (2009).
48. DeKosky, S. T. \& Scheff, S. W. Synapse loss in frontal cortex biopsies in Alzheimer's disease: correlation with cognitive severity. Ann. Neurol. 27, 457-464 (1990).

49. Ishizuka, Y. et al. Histone deacetylase mediates the decrease in drebrin cluster density induced by amyloid beta oligomers. Neurochem. Int. 76, 114-121 (2014).

50. Liu, D. et al. Targeting the HDAC2/HNF-4A/miR-101b/AMPK pathway rescues tauopathy and dendritic abnormalities in Alzheimer's disease. Mol. Ther. J. Am. Soc. Gene Ther. 25, 752-764 (2017).

51. Hearing, M., Graziane, N., Dong, Y. \& Thomas, M. J. Opioid and psychostimulant plasticity: targeting overlap in nucleus accumbens glutamate signaling. Trends Pharmacol. Sci. 39, 276-294 (2018).

52. Guegan, T., Cebria, J. P., Maldonado, R. \& Martin, M. Morphine-induced locomotor sensitization produces structural plasticity in the mesocorticolimbic system dependent on CB1-R activity. Addict. Biol. 21, 1113-1126 (2016).

53. Pal, A. \& Das, S. Chronic morphine exposure and its abstinence alters dendritic spine morphology and upregulates Shank1. Neurochem. Int. 62, 956-964 (2013).

54. Avey, D. et al. Single-cell RNA-seq uncovers a robust transcriptional response to morphine by glia. Cell Rep. 24, 3619-3629.e3614 (2018).

55. Ma, B. et al. L-stepholidine, a natural dopamine receptor D1 agonist and D2 antagonist, inhibits heroin-induced reinstatement. Neurosci. Lett. 559, 67-71 (2014).

56. Chandra, R. et al. Drp1 mitochondrial fission in D1 neurons mediates behavioral and cellular plasticity during early cocaine abstinence. Neuron 96, 1327-1341 e1326 (2017).

57. Sun, H. S. et al. BAZ1B in nucleus accumbens regulates reward-related behaviors in response to distinct emotional stimuli. J. Neurosci. 36, 3954-3961 (2016).

58. Paxinos, G. \& Watson, C. The Rat Brain in Stereotaxic Coordinates. (Elsevier Academic Press, Burlington, 2005).

59. Maze, I. et al. Essential role of the histone methyltransferase G9a in cocaineinduced plasticity. Science 327, 213-216 (2010).

60. Neve, R. L., Neve, K. A., Nestler, E. J., William, A. \& Carlezon, J. Use of herpes virus amplicon vectors to study brain disorders. Biotechniques 39, 381-391 (2005).

61. Geraldo, S., Khanzada, U. K., Parsons, M., Chilton, J. K. \& Gordon-Weeks, P R. Targeting of the F-actin-binding protein drebrin by the microtubule plustip protein EB3 is required for neuritogenesis. Nat. Cell Biol. 10, 1181-1189 (2008).

62. Hamilton, P. J. et al. Cell-type-specific epigenetic editing at the Fosb gene controls susceptibility to social defeat stress. Neuropsychopharmacol.: Off. Publ. Am. Coll. Neuropsychopharmacol. 43, 272-284 (2018).

63. Friedman, A. K. et al. Enhancing depression mechanisms in midbrain dopamine neurons achieves homeostatic resilience. Science 344, 313-319 (2014).

64. Sanz, E. et al. Fertility-regulating Kiss1 neurons arise from hypothalamic POMC-expressing progenitors. J. Neurosci.: Off. J. Soc. Neurosci. 35 , 5549-5556 (2015).

65. Loweth, J. A. et al. Synaptic depression via mGIuR1 positive allosteric modulation suppresses cue-induced cocaine craving. Nat. Neurosci. 17, 73-80 (2014).

66. Li, J. X., Thorn, D. A., Qiu, Y., Peng, B. W. \& Zhang, Y. Antihyperalgesic effects of imidazoline $\mathrm{I}(2)$ receptor ligands in rat models of inflammatory and neuropathic pain. Br. J. Pharmacol. 171, 1580-1590 (2014).

67. Siemian, J. N., Obeng, S., Zhang, Y., Zhang, Y. \& Li, J. X. Antinociceptive interactions between the imidazoline I2 receptor agonist 2-BFI and opioids in rats: role of efficacy at the mu-opioid receptor. J. Pharmacol. Exp. Ther. 357, 509-519 (2016).

68. Zachariou, V. et al. Essential role for RGS9 in opiate action. Proc. Natl Acad. Sci. USA 100, 13656-13661 (2003).

69. Renthal, W. et al. Genome-wide analysis of chromatin regulation by cocaine reveals a role for sirtuins. Neuron 62, 335-348 (2009).

70. Yuen, E. Y. et al. Repeated stress causes cognitive impairment by suppressing glutamate receptor expression and function in prefrontal cortex. Neuron 73, 962-977 (2012).

\section{Acknowledgements}

We would like to thank W. M. Hoffmann for technical assistance in actin assays. We thank S. McKnight for providing the RiboTag construct. We acknowledge the assistance of the Confocal Microscopy and Flow Cytometry Facility in the University at Buffalo Jacobs School of Medicine and Biomedical Sciences. This work was supported by the National Institutes of Health (no. NIDA R01DA037257 to D.M.D., NIDA R01DA037257-S1 to D.M.D., NIDA R21DA044486 to D.M.D., NIDA R01DA046818 to D.M.D., NIDA R01DA038613 to M.K.L., NIDA R01DA037618 to Z.Y., NINDS 
F99NS108543 to J.A.M., and NIGMS R25GM09545902 to University at Buffalo). The heroin and morphine used in these studies was generously gifted by the NIDA Drug Supply Program.

\section{Author contributions}

J.A.M., C.T.W., S.M., P.N., Z.-J.W., J.N.S., D.H., and A.M.G. performed the behavioral experiments. J.A.M., C.T.W., Z.-J.W., R.C., and M.L.K. conducted all Western blotting, mRNA, pull-down, and chromatin immunoprecipitation experiments. J.A.M., A.F.S., J.Z., K.E., and L.E.M. conducted the dendritic spine experiments. J.A.M. and P.Z. conducted the electrophysiology experiments. R.L.N., R.C., anD M.L.K. generated and provided the viral constructs. J.A.M., C.T.W., J.-X.L., A.M.G., Z.Y., and D.M.D. designed the experiments and analyzed the data. J.A.M., C.T.W., K.C.D., and D.M.D. wrote the manuscript.

\section{Additional information}

Supplementary Information accompanies this paper at https://doi.org/10.1038/s41467019-12122-8

Competing interests: The authors declare no competing interests.

Reprints and permission information is available online at http://npg.nature.com/ reprintsandpermissions/
Peer review information Nature Communications thanks the anonymous reviewers for their contribution to the peer review of this work.

Publisher's note Springer Nature remains neutral with regard to jurisdictional claims in published maps and institutional affiliations.

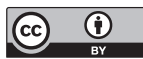

Open Access This article is licensed under a Creative Commons Attribution 4.0 International License, which permits use, sharing, adaptation, distribution and reproduction in any medium or format, as long as you give appropriate credit to the original author(s) and the source, provide a link to the Creative Commons license, and indicate if changes were made. The images or other third party material in this article are included in the article's Creative Commons license, unless indicated otherwise in a credit line to the material. If material is not included in the article's Creative Commons license and your intended use is not permitted by statutory regulation or exceeds the permitted use, you will need to obtain permission directly from the copyright holder. To view a copy of this license, visit http://creativecommons.org/ licenses/by/4.0/.

(C) The Author(s) 2019 